\title{
Resolution of electric field distribution and conductivity in a non- uniform electric field: A teaching proposal
}

\author{
Mustafa Erol ${ }^{\text {a }}$, ildahan Özdeyiş Çolak ${ }^{\text {b }}$ \\ Dokuz Eylül University, Cumhuriyet Blv, No:144, 35220, Alsancak, Konak, İzmir, Turkey \\ a mustafa.erol@deu.edu.tr, b ozdeyiscolak@gmail.com \\ * Corresponding Author.
}

Received: 10 July 2020; Revised: 13 December 2020; Accepted: 4 January 2021

\begin{abstract}
This work offers an alternative teaching proposal for the instruction of challenging concepts of electric field distribution and specific conductivity in a nonuniform electric field. Specifically, electric field lines are initially plotted and later on the relation between the electric potential difference and electric field strength is validated. Additionally, on a selected electric field line, electric field strength versus path length and also conductivity versus path length are plotted to comprehend and teach exceedingly difficult concepts of uniform and non-uniform electric fields. In order to accomplish those tasks, a basic conducting sheet, that is simply a wet cardboard, is designed as a part of the apparatus together with a dc power supply, a multi meter and connecting cables. The established method is interesting in the sense that designed the conducting wet cardboard is novel, very practical, beneficial and minimal costing, hence the approach offers physics educators fresh teaching routes and opportunities to clarify the puzzling concepts of electrical field and conductivity.

Keywords: physics education, non-uniform electric field, non-uniform conductivity, electric field line, electric field distribution, teaching electricity.
\end{abstract}

How to Cite: Erol, M., \& Çolak, İ. Ö. (2021). Resolution of electric field distribution and conductivity in a non-uniform electric field: A teaching proposal. Momentum: Physics Education Journal, 5(1), 1-9. https://doi.org/10.21067/mpej.v5i1.4576

\section{Introduction}

Physics studies the natural phenomena experimentally and theoretically, through complicated concepts and mathematical equations connecting those concepts. Physics Education Research (PER), on the other hand, aims to teach those concepts, related phenomena and mathematical equations with an enhanced comprehension. Physics educators, around the world, try to ease and teach intricate and so believed abstract concepts and equations such that every single student accomplishes conceptual understanding and internalizes the actual scientific knowledge (Retnawati et al., 2018; Zhang et al., 2018). Therefore, scientists resolve the natural phenomena by using tools such as graphs, charts, diagrams and eventually reach to mathematical equations to represent definite physical laws or principles (Brewe \& Sawtelle, 2018).

In this sense, electricity and magnetism are, for the majority of the students, believed to be abstract and puzzling subjects, henceforth need more contemplation and determination for an enriched understanding (Afra et al., 2009; Hand et al., 2009; Moodley \& Gaigher, 2019; Taber et al., 2006). Thus, teaching electricity and magnetism necessities more inspiring environments and ought to be handled more intelligently (Borges \& Gilbert, 1999; Gunstone et al., 2009; Kelly et al., 1998; Martinez et al., 2011). The concept of field, in physics, is substantially central and teaching of any field is challenging for the educators (Chazbeck \& Ayoubi, 2018; Furió \& Guisasola, 1998). Teaching electrical field and electrical conductivity, in a non-uniform electric field, is a more challenging task due to be containing continuously varying electric fields and conductivities. Especially electric field 
lines are believed to be abstract and majority of the students assume that there is no basic way to visualize the field lines (Ayars, 1996; Phillips et al., 2017). Uniform electric field lines are relatively easy to visualize, however non uniform electric field distribution and electric field lines are more difficult to visualize and to draw (Lincoln, 2017). The equipotential surfaces in 3D and equipotential lines in 2D are commonly used to teach the electric field distribution nevertheless finding an appropriate teaching material is a common problem (Rediansyah et al., 2015; Young, 2001). Additionally, instruction of the mathematical equation between the electric field vector and the electrical potential difference, in a non-uniform electric field, have been a confusing task for physics educators and teachers (Carlton, 1999; Cohen et al., 1983).

The present work specifically focuses on resolving apparent complications on instructing the intricate concepts of electric field distribution and conductivity in a non-uniform electric field. Consequently, present effort aims to develop a basic 2D material to demonstrate and teach the confusing electrical concepts. Following the development of the teaching material, electric field lines are initially drawn and the path length dependence of the electric field and also specific conductivity are determined. Additionally, the developed material is also employed to verify the mathematical relation between the electrical potential difference and the actual electric field strength on selected electric field lines. The present material is very advantageous and indeed novel in the sense that it costs minimal and works exceptionally well.

\section{Method}

\section{Research Model and Research Questions}

The present research focuses on developing novel teaching materials that could easily be employed within the basic physics laboratories with minimal costing. Therefore, the research approach can be classified as the descriptive approach and the research model can be named as developmental research model.

The work principally aims to resolve and teach the concepts of uniform and non-uniform electric fields, electric field lines, potential difference and specific conductivity in a no- uniform electric field, therefore following problem statements are formulized and clearly answered to reach the proposed goals of the study.

1. How can we visualize the electric field lines on a $2 D$ conducting sheet in a non-uniform electric field distribution?

2. How can we validate the equation of, $\Delta V=E \Delta x \cos \theta$, on an electric field line in a nonuniform electric field, by using $2 \mathrm{D}$ conducting sheet?

3. How does the magnitude of an electrical field change as a function of the path length on an electric field line in a non-uniform electric field?

4. How can one mathematically model the magnitude of an electrical specific conductivity change, as a function of the path length on an electric field line in a non-uniform electric field?

Teaching Material

One of the fundamental aims of this work was, in fact, to develop a 2D material that could be present in any school around the globe with minimal costing and easy to use. To manage this task, following a number of failures a simple smooth wet cardboard is finally employed in the design. The cardboard has a thickness of about $1 \mathrm{~mm}$ and dimensions of $28 \mathrm{~cm} \times 36 \mathrm{~cm}$. The cardboard, when dry, is a good insulator depending on its cellulosic nanostructure and could not be employed for the teaching purposes since no electric field and potential difference could be measured across. Knowing the fact that the water is actually a good electrical conductor made us to combine the cardboard with water. Therefore, dry smooth cardboard is simply immersed in an appropriate crystal clear tap water tank for about 5 seconds and finally a homogeneously distributed water molecules are obtained within the wet cardboard. Obviously, application of an electrical voltage more than the ionic bond energy for the water molecules, that is about $0,236 \mathrm{~V}$ per molecule, simply breaks the ionic bond between the hydrogen and hydroxide ions, $\mathrm{H}+$ and $\mathrm{OH}-$, and let them free such that they can move 
similarly to electrons and creates a non- uniform electric filed and conductivity. In the experiment, the evenly wet cardboard is just used as an opportunity to teach the crucial electrical concepts and hence chemical properties of the water such as distillation is not within the scope of the work.

\section{Experimental Details}

The apparatus employed for the work is shown in the Figure 1. The experimental setup is made up of a homogeneously wet cardboard, a DC power supply (max.18 V), a number of connection cables and a basic multi meter. During the actual experiment, a DC voltage of $15 \mathrm{~V}$ is applied to the two ends of the wet cardboard, however due to the contact problems, a DC voltage of $10.18 \mathrm{~V}$ is measured between the two ends of the cardboard or measurement points. The apparent difference is attributed to the well-known and unavoidable contact problem and does not affect the proposed teaching activities by any means.

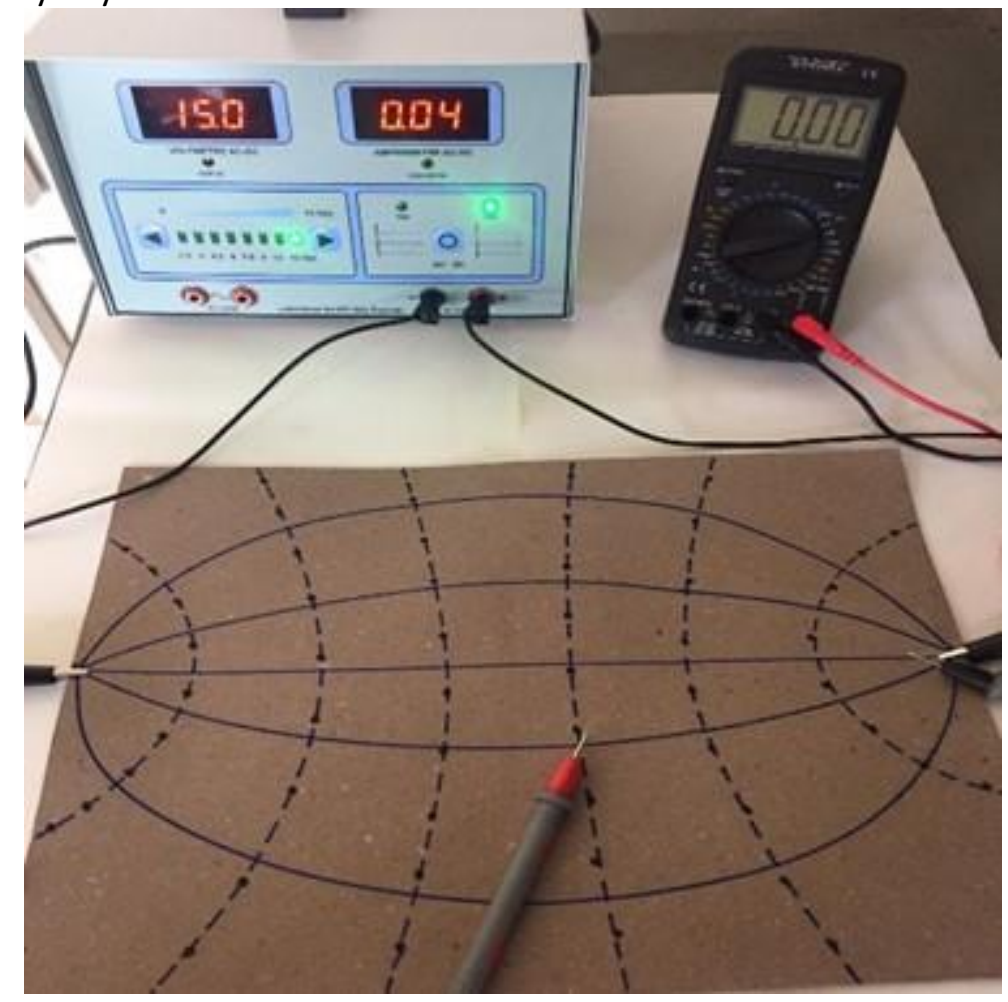

Figure 1. The photography of the apparatus, comprised of the developed wet conducting cardboard, a dc power supply, a multi meter and connecting cables

In order to answer the research problem statements following procedure is accomplished. For the first problem statement, basically the equipotential lines initially plotted for certain voltages and by using them the electric field lines are plotted perpendicularly. To answer the second problem statement, on selected electric field lines and on a specific point the potential difference is measured for $1 \mathrm{~cm}$ path lengths, for $\theta=0$ and $\theta=45$ and the proposed mathematical relation is maintained. For the third problem statement, on a selected electric field line, simply the voltage differences are sequentially measured, across the path intervals of $1 \mathrm{~cm}$, which is equal to the electric field strength. To answer the fourth problem statement, the electric field versus path length relation, obtained for the problem three, is simply employed and relevant specific conductivities are calculated, by using the equation of $I=\sigma E$.

\section{Results and Discussions}

\section{Visualizing non-uniform electric field lines on a conducting sheet}

In order to answer the first problem case, that is the visualizing the electric field lines in a nonuniform electric field distribution, initially equipotential points are determined for an appropriate 
voltages and distances. The appropriate voltage values are, 2,70 V, 4,70V, 5,23V, 6,37V, 7,24V and $8,69 \mathrm{~V}$, which are shown in the Figure 2 . This task is managed by fixing one probe at one end of the conducting paper and the second probe is used to search and find the equipotential points. Determined equipotential points are then followed to draw the equipotential curves. It is well known fact that the electric field lines ought to be perpendicular to the equipotential curves. Therefore, the electric field lines are straightforwardly plotted by a flexi curve ruler on the wet cardboard and the resultant curves are drawn by solid lines and shown in the Figure 2 (Lincoln, 2017).

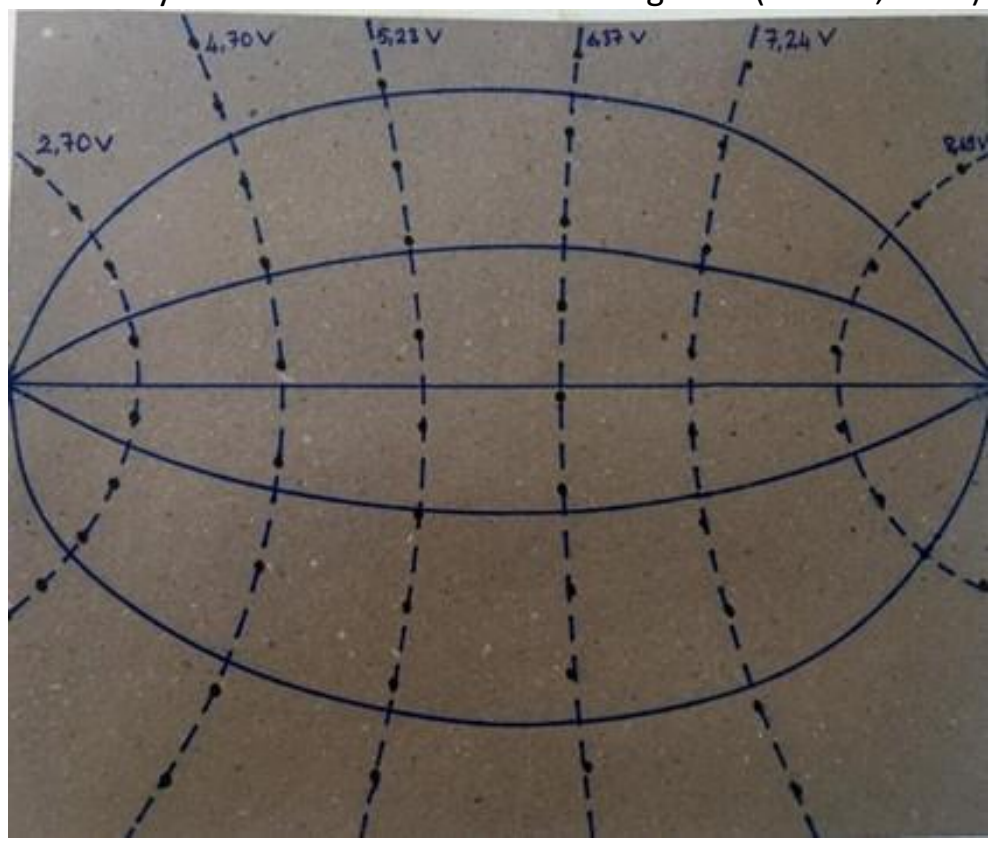

Figure 2. Experimentally measured equipotential lines shown by dotted lines and accordingly plotted perpendicular electric field lines shown by solid lines all plotted in a non-uniform electric field created on wet conducting cardboard

Validation of the potential difference equation in a non-uniform electric field

The second problem case was about the justification of the fundamental equation of, $\Delta V=E \Delta \mathrm{x} \cos \theta$, in a non-uniform electric field distribution (Beichner \& Serway, 2000). In the expression, $\theta$ denotes the angle between the actual electric field and the displacement vector and the task is achieved by two steps. The first step was managed by measuring the potential difference across $1 \mathrm{~cm}$ path lengths, $(\Delta x=1 \mathrm{~cm})$, on selected electric field line at a fixed point. The angle in this case is obviously zero, $(\theta=0)$ hence the measured voltage is directly equal to the electric field in terms of $\mathrm{V} / \mathrm{cm}\left(\Delta V=E_{0}\right)$ and this is named as as $\boldsymbol{E}_{0}$. In the second step, for the same point, the potential difference again across $1 \mathrm{~cm}$ long paths $(\Delta x=1 \mathrm{~cm})$ is measured for a fixed angle, $\theta=45^{\circ}$, in this case the voltage is equal to the $\mathrm{E},(\Delta V=\mathrm{E})$ In order to validate the actual equation, the expression of, $\cos \theta=\frac{E_{0}}{E}$, is executed and the outcomes are given in the Table 1. This procedure is carried out for five different points selected on various electric field lines which are schematically shown in the Figure 3 (Chazbeck \& Ayoubi, 2018).

The outcomes are obviously be compared with the theoretical value of, $\cos 45=0,707$ and relative error outcomes are also calculated and presented in the Table 1.

Path dependence of the electric field strength in a non-uniform electric field

The third problem situation focuses on the variation of the actual electric field strength on a selected electric field line. This specific task is managed by sequentially measuring the voltage differences across a path interval of $1 \mathrm{~cm}$. Since, on an electric field line, the electric field vector and the path/displacement vector are parallel to each other, then $\cos \theta=1$ and the measured voltage is directly equal to the electric field strength in $\mathrm{V} / \mathrm{cm}$ that is, $\Delta V=E \Delta x$. The outcomes of the measure- 
ments are tabulated in the Table 2. In the Table 2 calculated specific conductivity values are also given and to be used for the fourth problem case.

In order to investigate the mathematical relation between the path length and the magnitude of the electric field, the graph in between the mentioned variables is plotted and shown in the figure 4. The plot demonstrates a reasonably smooth and symmetric relation and mathematically the relation is given by $\mathrm{E}=0,0759 \mathrm{x}^{2}-0,4353 \mathrm{x}+1,2555$ in $\mathrm{V} / \mathrm{cm}$. A second order polynomial curve fit seems to be fitting well. This investigation clearly shows the non-uniform electric field distribution which basically originates from the non-uniform voltage distribution throughout the entire conducting sheet (Sears et al., 1987). The electrical voltage applied to the connection points of the conducting sheet was about $\mathrm{V}=15,00 \mathrm{~V}$, however the due to the unavoidable contact problems the actual applied voltage was about $V=10,18 \mathrm{~V}$. Specifically at the starting point of the path, the point $A$ and $x=0 \mathrm{~cm}$, the voltage was about $V=0,00 \mathrm{~V}$ and at the end of the path that is, the point $B$ and $x=35,5 \mathrm{~cm}$, the voltage was about $\mathrm{V}=10,18 \mathrm{~V}$.

The path length, $x$; dependence of the electric field strength, $E$, indicates that linearly varying charge density along the electric field line. This means that the charge density varies with the distance, $\mathrm{x}$, in accordance with, $\rho(x)=A x-B$, where $A$ and $B$ are constants. Considering this assumption and substitution of the equation within the well-known Poisson's equation of, $\vec{\nabla} \vec{E}=\frac{\rho(x)}{s}$, simply leads to the final result of, $E=A x^{2}-B x+C$, that is perfectly in agreement with the experimental outcome shown in the Figure 4. The approach (Figure 4) is quite expected since the positive ions, $\mathrm{H}^{+}$, would be accumulating around the negative potential point of $\mathrm{B}$ and the negative ions, $\mathrm{OH}^{-}$, would be accumulating around the positive potential point of $A$ of the wet cardboard (Retnawati et al., 2018).

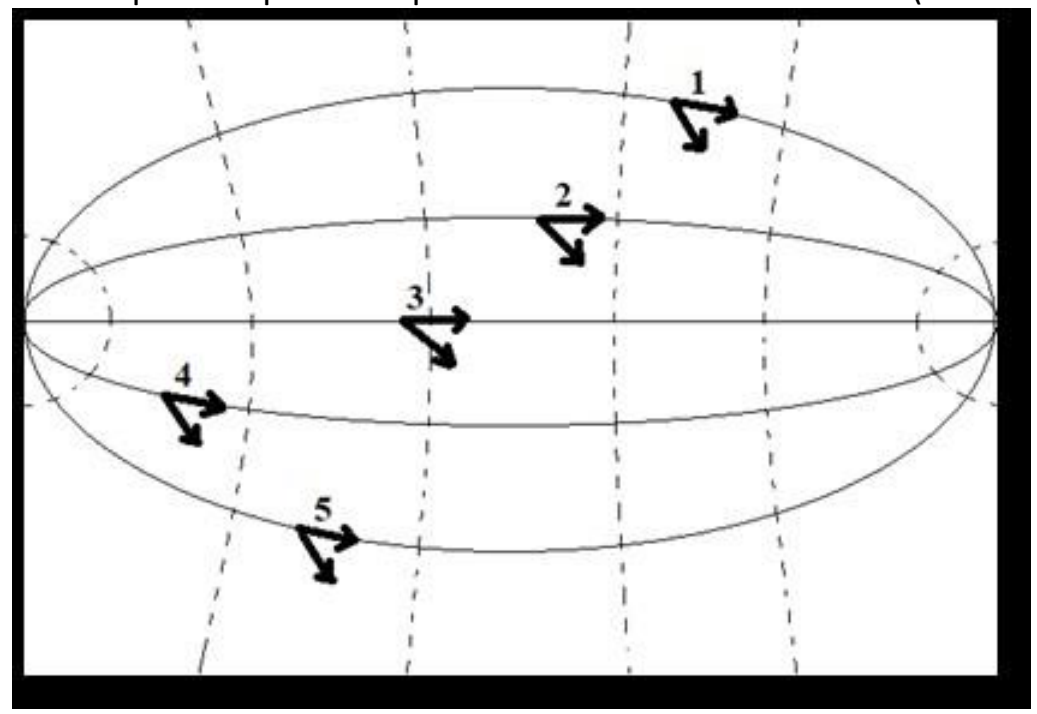

Figure 3. The measurement points numbered sequentially on the electric filed lines and used to measure the electric filed strengths for $\theta=0^{\circ}$ and for $\theta=45^{\circ}$, which are managed by measuring the voltages across $1 \mathrm{~cm}$ long paths

Table 1. Measurement results for the voltage differences across the $1 \mathrm{~cm}$ long path intervals on the electric field lines for $\theta=0^{\circ}$ and for $\theta=45^{\circ}$ together with the calculated $\cos \theta$ values and relative errors with respect to the theoretical value

\begin{tabular}{crrrc}
\hline Measurement point no & $E_{0}\left(V \mathrm{~cm}^{-1}\right)$ & $E\left(V c m^{-1}\right)$ & $\cos \theta$ & Relative Error (\%) \\
\hline 1 & 0,091 & 0,065 & 0,714 & 0,9 \\
2 & 0,072 & 0,049 & 0,680 & 3,8 \\
3 & 0,084 & 0,055 & 0,654 & 7,4 \\
4 & 0,082 & 0,056 & 0,682 & 3,5 \\
5 & 0,085 & 0,059 & 0,694 & 1,8 \\
\hline
\end{tabular}


Momentum: Physics Education Journal, 5 (1), 2021, 6

Mustafa Erol, i̇ldahan Özdeyiş Çolak

Table 2. Measurement results for the voltage differences across $1 \mathrm{~cm}$ long path intervals on a selected electric field line for $\theta=0^{\circ}$ together with the calculated specific conductivity values

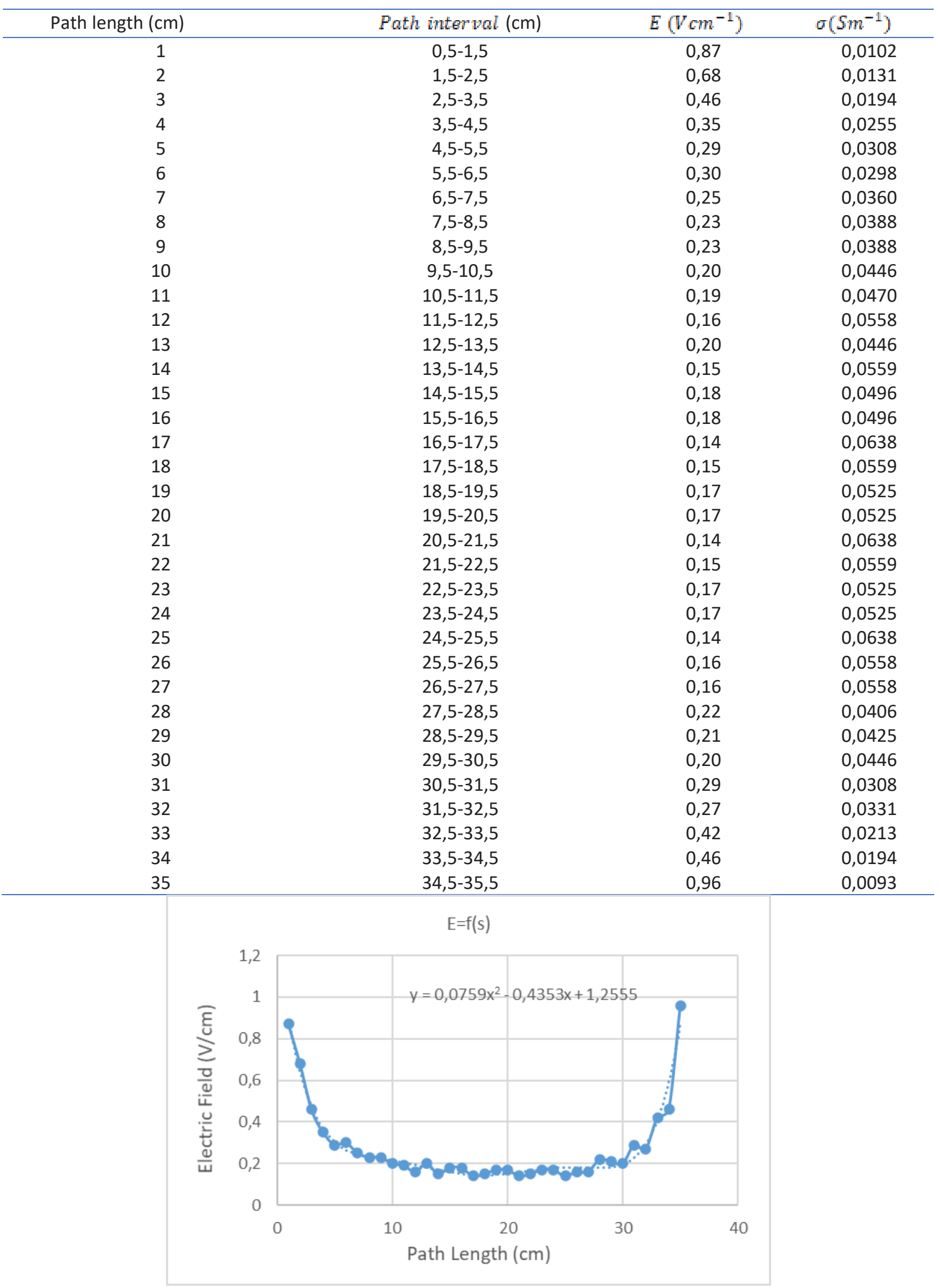

Figure 4. The measured potential difference values across a path length of $1 \mathrm{~cm}$ on a selected electric field line is plotted as a function of the sequential path length 


\section{Path dependence of the specific conductivity in a non-uniform electric field}

The electric field distribution on the wet cardboard primarily depends on the charge density distribution and is estimated previously. The specific conductivity distribution accordingly depends up on the charge density distribution and also the mean free time of the free charges according to the equation of, $(x)=\frac{\rho(x) e r(x)}{m}$. Nevertheless, the average current and also average current density can surely be employed to estimate the specific conductivity along an electric field line. Therefore, in order to answer the fourth problem statement, the well-known relation between the current density and the electric field strength, that is, $I=\sigma(x) E(x)$, is employed. The stable current is measured as, $\mathrm{I}=0,25 \mathrm{~mA}$, additionally the thickness of the conducting sheet was approximately $1 \mathrm{~mm}$ and the width was measured as $28 \mathrm{~cm}$. These specific numbers are used to calculate the actual conductivity and the outcomes of the calculation are given in the table 2 and plotted in the figure 5 .

The estimated specific conductivity is plotted as a function of the path length and the relation is mathematically found to be, $\sigma(x)=-0,159 x^{2}+0,5973 x+0,1756$, in $\mathrm{S} / \mathrm{m}$. The relation clearly shows higher conductivities as the electric field gets smaller, as expected, and this is surely due to the increase of the nobilities of the $\mathrm{H}^{+}$and $\mathrm{OH}^{-}$ions, assuming that there would be no change in the concentration of the freely moving ions. In other words, the $\mathrm{H}^{+}$and $\mathrm{OH}^{-}$ions experience less scattering as the electric field reduces which could also be a good example for non-ohmic conductivity for teaching purposes (Taber et al., 2006).

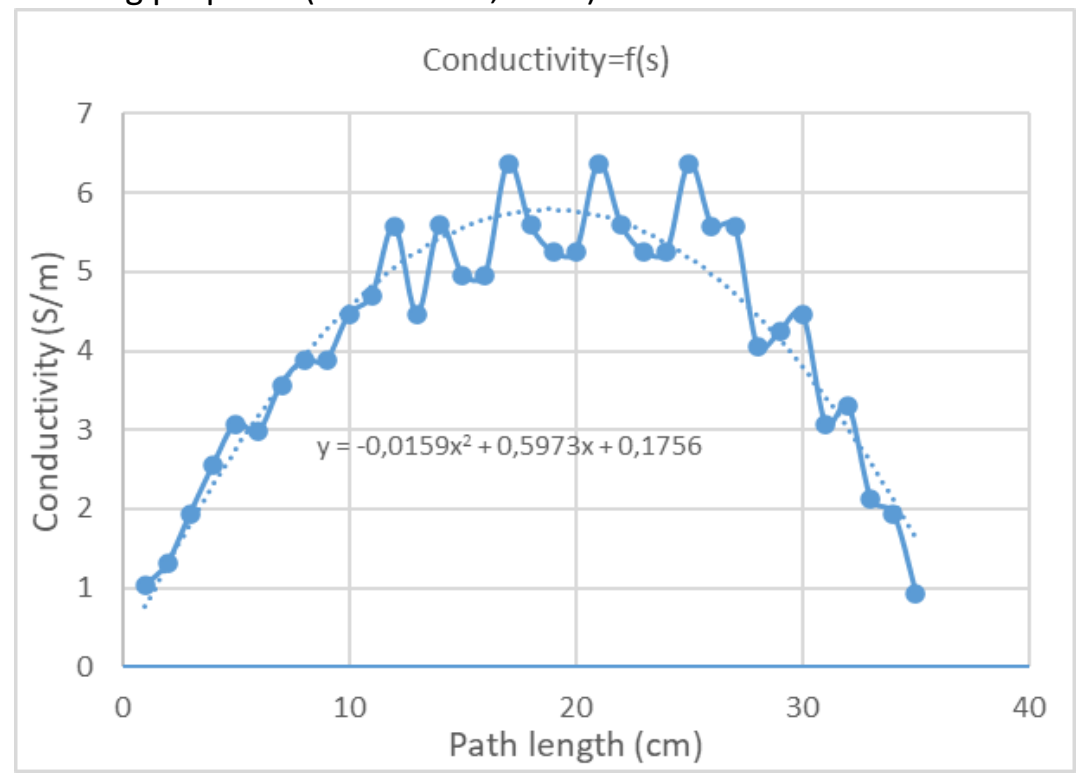

Figure 5. The specific conductivity values, calculated from the measured potential difference values across $1 \mathrm{~cm}$ path lengths on a selected electric field line, is plotted as a function of the sequential path length

\section{Conclusions}

The present work essentially offers a basic and costless accessible method to realize and teach the concepts of electric field, electric field line, electric potential difference and specific conductivity in a non-uniform electric field. The research questions are clearly answered one by one within the results section and the benefits of the present approach and the material are indeed clearly shown. The procedure outlined here basically employs a fresh novel conducting sheet which is only a wet smooth cardboard. The approach suggested is important due to developing a basic teaching material and outlines all the necessary information for teaching activities. The approach proposed presently is limited to only teaching activities, therefore any further insights concerning the details of the mobility of the charges and the effects of the actual cardboard material or detailed chemical properties of the water is beyond the scope of the work. However, we do believe that the method and the material offer a novel and powerful proposal for any physics educator. Especially, the conducting wet 
cardboard, that is very reachable and easy to use material, makes a great advantage for the students and indeed teachers to create a rich and beneficial teaching atmosphere.

\section{References}

Afra, N. C., Osta, I., \& Zoubeir, W. (2009). Students' alternative conceptions about electricity and effect of inquiry-based teaching strategies. International Journal of Science and Mathematics Education, 7(1), 103-132. https://doi.org/10.1007/s10763-007-9106-7

Ayars, E. (1996). Nonphysical results with the electric-field mapping experiment. The Physics Teacher, 34(6), 344-345. https://doi.org/10.1119/1.2344474

Beichner, R. J., \& Serway, R. A. (2000). Physics for scientists and engineers with modern physics. Saunders College Publishing.

Borges, A. T., \& Gilbert, J. K. (1999). Mental models of electricity. International Journal of Science Education, 21(1), 95-117. https://doi.org/10.1080/095006999290859

Brewe, E., \& Sawtelle, V. (2018). Modelling instruction for university physics: examining the theory in practice. European Journal of Physics, 39(5), 054001. https://doi.org/10.1088/13616404/aac236

Carlton, K. (1999). Teaching electric current and electrical potential. Physics Education, 34(6), 341345. https://doi.org/10.1088/0031-9120/34/6/401

Chazbeck, B., \& Ayoubi, Z. (2018). Resources used by lebanese secondary physics teachers' for teaching electricity: Types, objectives and factors affecting their selection. Journal of Education in Science, Environment and Health, 4(2), 118-128. https://doi.org/10.21891/jeseh.409487

Cohen, R., Eylon, B., \& Ganiel, U. (1983). Potential difference and current in simple electric circuits: A study of students' concepts. American Journal of Physics, 51(5), 407-412. https://doi.org/10.1119/1.13226

Furió, C., \& Guisasola, J. (1998). Difficulties in learning the concept of electric field. Science Education, 82(4), 511-526. https://doi.org/10.1002/(SICI)1098-237X(199807)82:4<511::AID-

SCE6 $>3.0 . C O ; 2-E$

Gunstone, R., Mulhall, P., \& McKittrick, B. (2009). Physics teachers' perceptions of the difficulty of teaching electricity. Research in Science Education, 39(4), 515-538. https://doi.org/10.1007/s11165-008-9092-y

Hand, B., Gunel, M., \& Ulu, C. (2009). Sequencing embedded multimodal representations in a writing to learn approach to the teaching of electricity. Journal of Research in Science Teaching, 46(3), 225-247. https://doi.org/10.1002/tea.20282

Kelly, G. J., Druker, S., \& Chen, C. (1998). Students' reasoning about electricity: combining performance assessments with argumentation analysis. International Journal of Science Education, 20(7), 849-871. https://doi.org/10.1080/0950069980200707

Lincoln, J. (2017). Electric field patterns made visible with potassium permanganate. The Physics Teacher, 55(2), 74-75. https://doi.org/10.1119/1.4974114

Martinez, F., Herrero, L. C., \& de Pablo, S. (2011). Project-based learning and rubrics in the teaching of power supplies and photovoltaic electricity. IEEE Transactions on Education, 54(1), 87-96. https://doi.org/10.1109/TE.2010.2044506

Moodley, K., \& Gaigher, E. (2019). Teaching electric circuits: teachers' perceptions and learners' misconceptions. Research in Science Education, 49(1), 73-89. https://doi.org/10.1007/s11165017-9615-5

Phillips, J. A., Sanny, J., Berube, D., \& Hoemke, A. (2017). Beyond the point charge: equipotential surfaces and electric fields of various charge configurations. The Physics Teacher, 55(2), 71-73. https://doi.org/10.1119/1.4974113

Rediansyah, H., Khairurrijal, \& Viridi, S. (2015). Static electric field mapping using a mosquito racket 
and baby oil. Physics Education, 50(6), 690-693. https://doi.org/10.1088/0031-9120/50/6/690

Retnawati, H., Arlinwibowo, J., Wulandari, N., \& Pradani, R. (2018). Teachers' difficulties and strategies in physics teaching and learning that applying mathematics. Journal of Baltic Science Education, 17(1), 120-135. http://www.scientiasocialis.lt/jbse/?q=node/643

Sears, F. W., Zemansky, M. W., \& Young, H. D. (1987). University physics. Addison-Wesley.

Taber, K. S., Trafford, T. de, \& Quail, T. (2006). Conceptual resources for constructing the concepts of electricity: the role of models, analogies and imagination. Physics Education, 41(2), 155-160. https://doi.org/10.1088/0031-9120/41/2/006

Young, R. A. (2001). Quantitative experiments in electric and fluid flow field mapping. American Journal of Physics, 69(12), 1223-1230. https://doi.org/10.1119/1.1405508

Zhang, C., Wang, H., Liu, Y., \& Jiang, J. (2018). Investigation and the improvement strategy of the inquiry physics experiment teaching in senior high school. American Journal of Physics and Applications, 6(5), 104. https://doi.org/10.11648/j.ajpa.20180605.11 